\title{
Assessing Early Brain Development in Neonates by Segmentation of High-Resolution 3T MRI
}

\author{
Guido Gerig $^{1,2}$, Marcel Prastawa ${ }^{1}$, Weili Lin ${ }^{3}$, and John Gilmore ${ }^{2}$ \\ 1 Departments of Computer Science, \\ 2 Psychiatry, \\ 3 Radiology \\ University of North Carolina, Chapel Hill, NC 27599, USA \\ gerig@cs.unc.edu ${ }^{\dagger}$
}

\begin{abstract}
This paper describes effort towards automatic tissue segmentation in neonatal MRI. Extremely low contrast to noise ratio (CNR), regional intensity changes due to $\mathrm{RF}$ coil inhomogeneity and biology, and tissue property changes due to the early myelination and axon pruning processes require a methodology that combines the strength of spatial priors (template atlas), data modelling, and prior knowledge about brain development. We use an EM-type algorithm that includes tissue classification, inhomogeneity correction and brain stripping into an iterative optimization scheme using a mixture distribution model. A statistical brain atlas registered to the subject image serves as a spatial prior. White matter in neonates is modeled as a mixture model of non-myelinated and myelinated regions. A pilot study on 10 neonates demonstrates the feasibility of high-resolution neonatal MRI and of automatic tissue segmentation. Results demonstrate that interleaved segmentation and inhomogeneity correction, guided by a statistical spatial prior, will provide a powerful and efficient segmentation framework for this type of imaging data. It is demonstrated that the mixture model for white matter allows us to segment early myelination regions of the projection tract up to the motor cortex, while also providing non-myelinated white, gray and csf segmentation. The early myelination regions are hypothesized to develop early but have not yet been shown in quantitative MRI studies.
\end{abstract}

Neonatal brain MRI segmentation is very challenging due to low contrast, tissue intensity variation, scanner bias field, lack of contrast, and fractional myelination of white matter. Results presented in [1] clearly demonstrate the difficulty as brain segmentation of 1 months old infant MRI mostly show noise although visual inspection of the MRI leaves the impression that there are discernable intensity differences for non-myelinated white and for gray matter. Most advanced work has been demonstrated by 23 , where subjects were mostly pre-term babies presenting less complex cortical folding. This method combined supervised voxel classification with a geometric model derived from a registered segmentation template. We have developed an integrated, automatic package for neonatal MRI segmentation which is closely related to the atlas-driven segmentation

\footnotetext{
$\dagger$ This research is supported by the UNC Neurodevelopmental Disorders Research Center HD 03110, the NIH Conte Center MH064065, the Stanley Medical Research Institute, and the Foundation of Hope. Color paper version: www.cs.unc.edu/ ${ }^{\sim}$ gerig.
} 
presented in 4. We created an atlas template of a 2-week old infant and an improved concept for estimation of the bi-modal distribution of myelinated versus non-myelinated tissue within white matter. Results on 10 subjects are very enrouraging and demonstrate feasibility. We could segment white matter, gray matter, csf and early myelination regions. In addition, we consistently found hyperintense cortical gray in the area of the motor cortex, suggesting that high-field $3 \mathrm{~T}$ MRI is a sensitive technique to study early brain development.
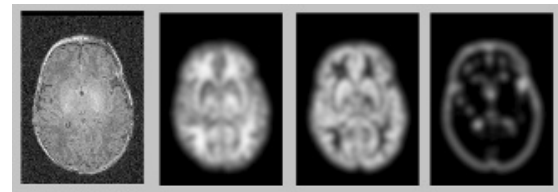

Fig. 1. Template MRI and blurred segmentation maps for white, gray and csf.
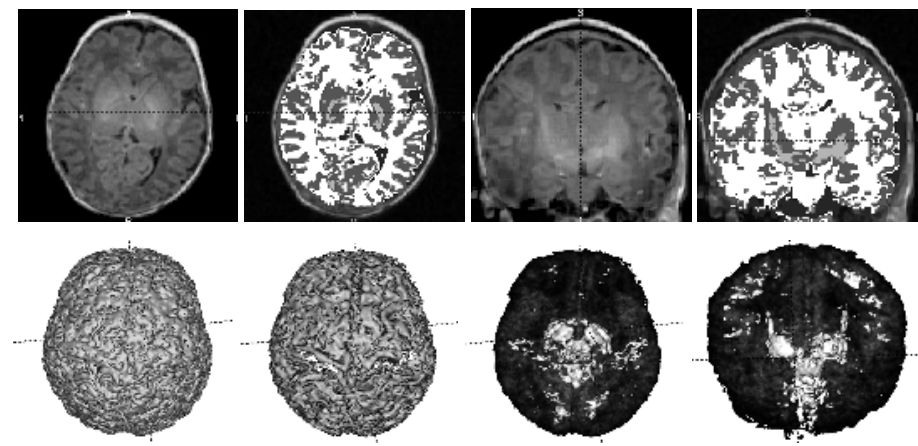

Fig. 2. Atlas-driven segmentation of neonate $\mathrm{MRI}$ (T1w, $1 \mathrm{~mm} 3$ resolution). Top: Axial and coronal MRI sections with corresponding classification maps (white-matter (white), gray matter (dark gray), csf (black), and early myelination regions (light grey). Bottom: 3D rendering of cortical surface, white matter surface, and early myelinated white and cortical gray matter structures (top and front view).

\section{References}

1. Matsuzawa, J., Matsui, M., Konishi, T., Noguchi, K., Gur, R., Bilder, W., Miyawaki, T.: Age-related volumetric changes of brain gray and white matter in healthy infants and children. Cerebral Cortex 11 (2001)

2. Warfield, S., Kaus, M., Jolesz, F., Kikinis, R.: Adaptive template moderated spatially varying statistical classification. In Wells, W.M.e.a., ed.: Medical Image Computing and Computer-Assisted Intervention (MICCAI'98). Volume 1496 of LNCS., Springer (1998)

3. Hüppi, P., Warfield, S., Kikinis, R., Barnes, P., Zientara, G., Jolesz, F., Tsuji, M., Volpe, J.: Quantitative magnetic resonance imaging of brain development in premature and normal newborns. Ann Neurol 43 (1998) 224-235

4. Van Leemput, K., Maes, F., Vandermeulen, D., Suetens, P.: Automated modelbased tissue classification of MR images of the brain. IEEE Transactions on Medical Imaging 18 (1999) 897-908 